\title{
New results for the Herzenberg dynamo: steady and oscillatory solutions
}

\author{
By A. Brandenburg ${ }^{1}$, D. Mosinand A. M. Soward ${ }^{3}$ \\ ${ }^{1}$ Department of Mathematics, University of Newcastle upon Tyne, Merz Court, \\ Newcastle upon Tyne NE1 YRU, UK \\ ${ }^{2}$ Department of Mathematics, University of Manchester, Oxford Road, \\ Manchester M13 9PL, UK \\ ${ }^{3}$ Department of Mathematics, University of Exeter, Laver Building, \\ North Park Road, Exeter EX 4 4QE, UK
}

Received 25 February 1997; accepted 30 July 1997

The Herzenberg dynamo, consisting of two rotating electrically conducting spheres with non-parallel spin axes, immersed in a finite spherical conducting medium, is simulated numerically for a variety of parameters not accessible to the original asymptotic theory. Our model places the spheres in a spatially periodic box. The largest growth rate is obtained when the angle, $\varphi$, between the spin axes is somewhat larger than $125^{\circ}$. In agreement with the asymptotic analysis, it is found that the critical dynamo number is approximately proportional to the cube of the ratio of the common radius of the spheres and their separation. The asymptotic prediction, strictly valid only in the limit of small spheres, remains approximately valid even when the diameter of the spheres becomes comparable to their separation. For $|\varphi|<90^{\circ}$ we also find oscillatory solutions, which were not predicted by Herzenberg's analysis. To understand such solutions we present a modified asymptotic analysis in which the separation of the two spheres is essentially replaced by the skin depth which, in turn, depends on the diameter of the spheres. The magnetic field consists of magnetic flux rings wrapped around the two spheres. Applications to local models of turbulent dynamos and to dynamo action in binary stars are discussed.

Keywords: Herzenberg dynamo; Reynolds number;

steady laminar flow; magnetic field strength

\section{Introduction}

Herzenberg (1958) produced one of the two first rigorous proofs of the existence of fluid dynamo action (see also Backus 1958). He considered two rotating spheres with non-parallel axes immersed in an electrically conducting medium, of spherical form, bounded by an insulator. His analysis was valid for the limit of small $a / d$, where $a$ is the radius of the spheres and $2 d$ their separation. Dynamo action is possible for about half the possible relative orientations of the spin axes of the spheres, and the fields are steady. Moffatt (1978) gives an accessible outline of the theory. Nevertheless, subtle issues emerge from the two rotor system, which stimulated the further analytic studies of Gibson (1968a) and Gailitis (1973) and was the focus of discussion in the reviews of Gibson \& Roberts (1967), Roberts (1967a,b), Gibson (1969) and Roberts (1971). Generalizations include multiple rotors like the three-rotor system of Gibson

Proc. R. Soc. Lond. A (1998) 454, 1283-1300

Printed in Great Britain
(C) 1998 The Royal Society

TEX Paper 
(1968b), although the idea of image rotors to accommodate boundary conditions in electromagnetic induction problems had earlier been introduced by Herzenberg \& Lowes (1957).

An experimental version of a similar dynamo, in the form of two rotating conducting cylinders, was constructed by Lowes \& Wilkinson (1963). Although their experiment was inspired by Herzenberg's result, it necessarily differed from his model in several significant details. The cylinders were embedded in a solid block of the same material, and electrical contact between the rotors and the block was provided by a thin lubricating film of mercury. They verified that dynamo action occurred, and in a subsequent paper (Lowes \& Wilkinson 1968) they found also non-steady behaviour for supercritical values of the angular velocities, when viscous dissipation was reduced. It is unclear whether the unsteady behaviour was due to the excitation of an unsteady linear dynamo mode, or to a nonlinearity caused by the experimental set-up.

Dolginov \& Urpin (1979) have since discussed applications to binary star systems but, to our knowledge, there has been little further investigation of the problem.

We describe here a numerical simulation of a Herzenberg-like dynamo in a finite box, that is not restricted to the limit of small $a / d$. We demonstrate that dynamo action occurs for values of $a / d$ between 0.5 and 0.9 , and that Herzenberg's results for the relative orientations of the spin vectors, for which steady dynamo action occurs, remain quite accurate even when $a / d$ is not small. Though Herzenberg's asymptotic results apply to an unbounded domain, they still provide a good approximation for large (but finite) box size. To improve the accuracy of the asymptotic theory relevant to our simulations, we consider the appropriate image system for our boundary conditions. This leads to a spatially periodic array of rotors in all three coordinate axis directions. In this context, we remark that Roberts (1971, p. 150) discussed briefly the case of a large number of identical rotors, and indicated how they can give rise to a version of mean field electrodynamics; the idea was developed further by Childress (1983). We also stress that the vector invariant approach, which we use in our analytic treatment, was introduced by Gibson \& Roberts (1967); it leads, as subsequent authors have realized, to considerable mathematical simplification and physical clarity. We also, for the first time, present a visualization of the magnetic field for this class of dynamo action. Further, a new class of oscillatory dynamo solutions is described, that exist for spin vector orientations for which steady dynamo action does not occur. Gailitis (1973) drew attention to the existence of oscillatory modes but only to resolve difficulties with low order effects within the range of orientations for which steady dynamo action occurs, and so only touched the periphery of the wider class that we identify.

\section{The model}

We consider two conducting spheres of radius $a$, separated by a distance $2 d$, spinning with angular velocities $\boldsymbol{\omega}_{1}$ and $\boldsymbol{\omega}_{2}$ in a medium with uniform conductivity $\eta$, equal to that of the spheres. In Herzenberg's original analysis the two spheres were embedded in a larger conducting sphere, outside of which a vacuum was assumed. In our numerical analysis we use a simple Cartesian geometry and embed the two spheres in a medium of uniform conductivity, with no exterior vacuum. The positions of the centres of the two spheres are

$$
\boldsymbol{x}_{1}=(-d, 0,0), \quad \boldsymbol{x}_{2}=(d, 0,0),
$$

Proc. R. Soc. Lond. A (1998) 
and their angular velocities are

$$
\boldsymbol{\omega}_{1}=\omega_{1}\left(0,-\frac{1}{2} \sin \varphi, \frac{1}{2} \cos \varphi\right), \quad \boldsymbol{\omega}_{2}=\omega_{2}\left(0, \frac{1}{2} \sin \varphi, \frac{1}{2} \cos \varphi\right),
$$

where $\omega_{1}$ and $\omega_{2}$ are specified below. Purely for numerical convenience, we make the boundaries of the spheres 'soft' so that velocity gradients remain finite, by adopting modified gaussian profiles,

$$
\omega_{1,2}(\boldsymbol{x})=\omega \exp \left(-\left[\left|\boldsymbol{x}-\boldsymbol{x}_{1,2}\right| / a\right]^{n}\right),
$$

with, fairly arbitrarily, $n=10$. The resulting (steady) velocity field is then

$$
\boldsymbol{u}(\boldsymbol{x})=\sum_{i=1,2} \boldsymbol{\omega}_{i} \times\left(\boldsymbol{x}-\boldsymbol{x}_{i}\right)
$$

The evolution of the magnetic field $\boldsymbol{B}$ is governed by the induction equation,

$$
\frac{\partial \boldsymbol{B}}{\partial t}=\nabla \times(\boldsymbol{u} \times \boldsymbol{B})+\eta \nabla^{2} \boldsymbol{B} .
$$

To ensure that the condition $\nabla \cdot \boldsymbol{B}=0$ is satisfied at all times we solve for the vector potential $\boldsymbol{A}$, where $\boldsymbol{B}=\nabla \times \boldsymbol{A}$. The gauge is chosen so that equation (2.5) yields $(\eta$ uniform)

$$
\frac{\partial \boldsymbol{A}}{\partial t}=\boldsymbol{u} \times(\nabla \times \boldsymbol{A})+\eta \nabla^{2} \boldsymbol{A}
$$

We integrate the equations numerically in a Cartesian box with periodic boundary conditions on $(x, y)= \pm L$, and impose the condition $B_{x}=B_{y}=0$ at $z= \pm L_{z}$, where $L_{z}=\frac{1}{2} L$ in most cases. (With this condition the mean horizontal magnetic field is not constrained to be zero; cf. Brandenburg et al. (1995). Nevertheless, in none of the cases studied below is a mean magnetic field is excited.) For $\omega=0$ the decay rate was found numerically to be close to the expected value of $2 \pi^{2} \eta / L^{2}$. By taking $d<\frac{1}{2} L$ the separation of the spheres within the computational domain is smaller than the separation across the periodic boundaries. Visualization of the magnetic field shows that when there is dynamo action the field is indeed concentrated near and in between the spheres, and so does not appear to result from, or to be affected by, the imposed periodicity.

We approximate derivatives on our Cartesian mesh by centred differences. We use sixth-order compact derivatives (Lele 1992) and a third-order scheme to advance the equations in time. Lengths and time are measured in units of $L$ and global diffusion time, $L^{2} / \eta$, respectively. The governing control parameters that we vary are $\varphi, a, d$ and the Reynolds number based on the radius of the spheres,

$$
R_{\mathrm{m}}=\omega a^{2} / \eta=(a / L)^{2} C_{\omega},
$$

The Reynolds number based on the scale $L, C_{\omega}=\omega L^{2} / \eta$, is also given for comparison.

Since equation (2.5) is linear in $\boldsymbol{B}$, the solution is of the form $\boldsymbol{B}(\boldsymbol{x}, t)=$ $\sum_{i=1}^{\infty} \boldsymbol{b}_{i}(\boldsymbol{x}) \exp \left(\lambda_{i} t\right)$, where the $\boldsymbol{b}_{i}(\boldsymbol{x})$ are eigenfunctions with eigenvalues $\lambda_{i}$, which are in general complex. In most cases we adopted as initial condition a ring field with $A_{x}=A_{y}=0$ and

$$
A_{z}=\exp \left[-\left(r / r_{0}\right)^{2}-\left(z / z_{0}\right)^{2}\right]
$$

where $r^{2}=x^{2}+y^{2}$ and $r_{0}=z_{0}=0.2$. After some time the eigenfunction with the largest value of $\operatorname{Re} \lambda_{1}$ will dominate. This is achieved when $\lambda(t) \equiv \mathrm{d} \ln \left\langle\boldsymbol{B}^{2}\right\rangle^{1 / 2} / \mathrm{d} t$ becomes constant, so that $\operatorname{Re} \lambda_{1}=\lim _{t \rightarrow \infty} \lambda(t)$. Here, the angular brackets denote a spatial average. If the solution is oscillatory, the average is also taken over one

Proc. R. Soc. Lond. A (1998) 
oscillation period, $2 \pi / \operatorname{Im} \lambda_{1}$. Although Herzenberg's original analysis was concerned with steady solutions, oscillatory solutions are also possible, as we will see below. In most cases the solution begins to grow or decay exponentially after about 0.2 diffusion times, although for decaying solutions the exponential decay can sometimes begin somewhat later.

\section{Results}

Herzenberg's analytical treatment for two rotors is only valid in the small sphere limit $a \ll d$. His results are thus only applicable to our configuration for the large box limit $d \ll L=O\left(L_{z}\right)$. This double limit is difficult to approach in the present numerical treatment. Still, his method does not rely on the latter limit and so we extend his analytic results to the case of finite box size in the Appendix by considering a suitable spatially periodic array of rotors in unbounded space. This analysis continues to assume $a / d \ll 1$.

Numerically, as a compromise with Herzenberg's original two rotor model, we take $a \lesssim d \lesssim \frac{1}{2} L$. We begin by investigating the optimal angle, $\varphi$, for the case $d / L=0.45$. At first we used $63 \times 63 \times 32$ meshpoints, but it turned out that a lower resolution of $31 \times 31 \times 16$ meshpoints gave growth rates that are very similar as at the higher resolution. Therefore we were able to perform a parameter survey using only $31 \times 31 \times 16$ meshpoints for many cases.

\section{(a) Dependence on the angle $\varphi$}

From Moffatt (1978, equation (6.79)), the square of the inverse critical Reynolds number for dynamo action in the two rotor system is given by

$$
R_{\mathrm{m}}^{-2}=-\frac{1}{4800}\left(\frac{a}{d}\right)^{6} \sin ^{2} \varphi \cos \varphi,
$$

a special case of the general result (Roberts, $1967 a$, p. 99, equation (99)) appropriate to more general orientations of the rotors. The maxima of $R_{\mathrm{m}}^{-2}$ occur in the second and third quadrants where $\tan \varphi=\sqrt{ } 2$, so $\varphi \approx \pm 125^{\circ}$. The modified form of equation (3.1), which accommodates the boundary conditions of our periodically finite domain, is given by equation (A 19).

In figure 1 we show the largest growth rate $\operatorname{Re} \lambda_{1}$ as a function of the angle $\varphi$ for $a=0.4, d=0.45, R_{\mathrm{m}}=480$. Note that the growth rate is positive for $95^{\circ} \lesssim|\varphi| \lesssim$ $165^{\circ}$. The maximum value of the growth rate is obtained for $\varphi$ a little larger than $125^{\circ}$ (the optimal asymptotic value)

\section{(b) Dependence on a and $d$}

In table 1 we present the marginal values of $R_{\mathrm{m}}$ (and $C_{\omega}$ ) above which dynamo action is possible, for the theoretically optimal angle $\varphi=125^{\circ}$. In the table we also give the maximum velocity and the 'mesh' Reynolds number, $R_{\mathrm{g}}=u_{\max } \delta x / \eta$, which is based on the mesh size and the maximum velocity. The numerical advection scheme becomes inaccurate if this number becomes too large. We showed empirically that the marginal value of $R_{\mathrm{m}}$ on a $63 \times 63 \times 32$ mesh ( $R_{\mathrm{g}}$ about 50$)$ differs by less than $1 \%$ from that found on a $31 \times 31 \times 16$ mesh $\left(R_{\mathrm{g}}\right.$ about 25$)$, and so certainly have confidence in the accuracy of our simulations when $R_{\mathrm{g}} \lesssim 100$. Given the overall consistency and uniformity of our results (below), we are reasonably happy even with the last entry of table 1 with $R_{\mathrm{g}}=158$.

Proc. R. Soc. Lond. A (1998) 


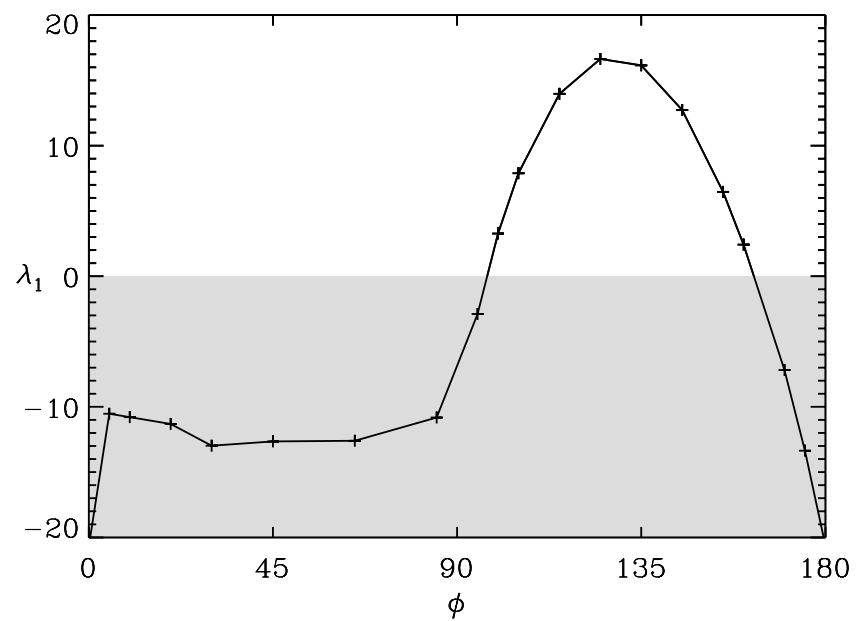

Figure 1. The largest growth rate $\lambda_{1}$ as a function of the angle $\varphi \cdot a=0.4, d=0.45, R_{\mathrm{m}}=480$, so $C_{\omega}=3000$. The negative growth rates (shaded area) are less well established than the positive growth rates, because it then takes longer for the solution to select the eigenfunction with the smallest decay rate.

Table 1. Marginal values of $C_{\omega}$ and $R_{\mathrm{m}}$ for $\varphi=125^{\circ}$

(The maximum flow velocity $u_{\max }$ and the mesh Reynolds number $R_{\mathrm{g}}$ are given for orientation.)

\begin{tabular}{|c|c|c|c|c|c|c|c|}
\hline resolution & $L_{z}$ & $a$ & $d$ & $C_{\omega}$ & $R_{\mathrm{m}}$ & $u_{\max }$ & $R_{\mathrm{g}}$ \\
\hline $31 \times 31 \times 16$ & $\frac{1}{2} L$ & 0.40 & 0.45 & 1490 & 239 & 0.29 & 27 \\
\hline $31 \times 31 \times 16$ & $\frac{1}{2} L$ & 0.35 & 0.45 & 3440 & 421 & 0.25 & 55 \\
\hline $63 \times 63 \times 32$ & $\frac{1}{2} L$ & 0.30 & 0.45 & 8570 & 771 & 0.22 & 59 \\
\hline $31 \times 31 \times 16$ & $\frac{1}{2} L$ & 0.35 & 0.40 & 1500 & 184 & 0.25 & 24 \\
\hline $31 \times 31 \times 16$ & $\frac{1}{2} L$ & 0.30 & 0.40 & 3740 & 337 & 0.22 & 51 \\
\hline $63 \times 63 \times 32$ & $\frac{1}{2} L$ & 0.30 & 0.40 & 3740 & 336 & 0.22 & 26 \\
\hline $31 \times 31 \times 32$ & $L$ & 0.30 & 0.40 & 3670 & 330 & 0.22 & 26 \\
\hline $63 \times 63 \times 32$ & $\frac{1}{2} L$ & 0.25 & 0.40 & 10120 & 633 & 0.18 & 58 \\
\hline $31 \times 31 \times 16$ & $\frac{1}{2} L$ & 0.30 & 0.35 & 1900 & 171 & 0.22 & 26 \\
\hline $63 \times 63 \times 32$ & $\frac{1}{2} L$ & 0.25 & 0.35 & 6000 & 375 & 0.18 & 34 \\
\hline $63 \times 63 \times 32$ & $\frac{1}{2} L$ & 0.20 & 0.35 & 18270 & 731 & 0.14 & 83 \\
\hline $63 \times 63 \times 32$ & $\frac{1}{2} L$ & 0.17 & 0.35 & 41600 & 1200 & 0.12 & 158 \\
\hline
\end{tabular}

We also checked the dependence of the results on the vertical extent $L_{z}$ of the box. We found that in the case $a=0.3, d=0.4$ the critical value of $R_{\mathrm{m}}$ is lowered by $2 \%$ when we double $L_{z}$ from $\frac{1}{2} L$ to $L$; see table 1 .

In figure 2 we plot the marginal value of $R_{\mathrm{m}}$ versus $a / d$ for different values of $a$ and $d$, when $\phi=125^{\circ}$. We also show the asymptotic result (3.1). The remaining discrepancy appears to be mostly due to the periodicity assumed in the numerical solutions, absent in the Herzenberg model. With due account taken of the spatial periodicity and vertical boundary conditions in the Appendix, table 2 shows that the discrepancies are significantly reduced.

Proc. R. Soc. Lond. A (1998) 
Table 2. Comparison of the asymptotic results (3.1) and (A 19) with the numerical results

\begin{tabular}{rcccccc}
\hline$a$ & $d$ & $L_{z}$ & $(3.1)$ & $(\mathrm{A} 19)$ & num. \\
\hline 0.40 & 0.45 & $\frac{1}{2} L$ & 159 & 281 & 239 \\
0.35 & 0.45 & $\frac{1}{2} L$ & 237 & 419 & 421 \\
0.30 & 0.45 & $\frac{1}{2} L$ & 377 & 665 & 771 \\
0.35 & 0.40 & $\frac{1}{2} L$ & 167 & 215 & 184 \\
0.30 & 0.40 & $\frac{1}{2} L$ & 265 & 341 & 336 \\
0.30 & 0.40 & $L$ & 265 & 338 & 330 \\
0.25 & 0.40 & $\frac{1}{2} L$ & 457 & 590 & 633 \\
0.30 & 0.35 & $\frac{1}{2} L$ & 177 & 200 & 171 \\
0.25 & 0.35 & $\frac{1}{2} L$ & 306 & 346 & 375 \\
0.20 & 0.35 & $\frac{1}{2} L$ & 599 & 676 & 731 \\
0.17 & 0.35 & $\frac{1}{2} L$ & 975 & 1101 & 1200 \\
\hline
\end{tabular}

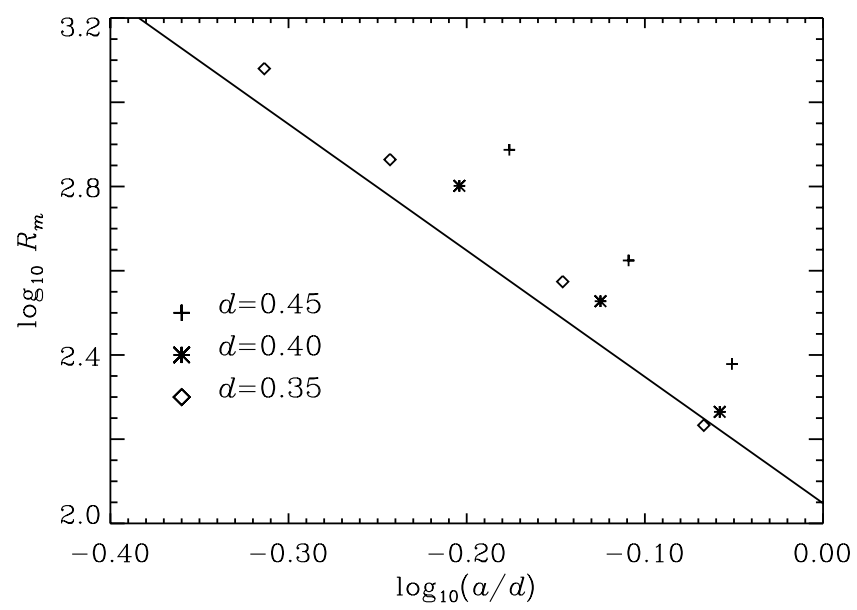

Figure 2. The marginal value of $R_{\mathrm{m}}$ versus $a / d$ for different values of $a$ and $d$, for $\varphi=125^{\circ}$.

The solid line represents the asymptotic solution (3.1), which has the slope -3 .

\section{(c) Field geometry}

We visualize the magnetic field by plotting the $\boldsymbol{B}$ vectors in three-dimensional space in those places where the magnetic field strength exceeds a certain threshold value; see figure 3 . Note that the field forms tube-like structures, with the $\boldsymbol{B}$-vectors aligned with the tubes.

There is a smaller flux ring near the tips of the $\boldsymbol{\omega}$ arrows with $\boldsymbol{B}$ parallel to $\boldsymbol{u}$, and near the tails of the arrows there is a stronger flux ring with $\boldsymbol{B}$ antiparallel to $\boldsymbol{u}$. This local reflectional asymmetry about each rotor's equator is predicted by Herzenberg's theory. Essentially, the dominant induced magnetic field is axisymmetric and toroidal. The lowest harmonic induced by the differential rotation acting on a dipole-like axisymmetric field is antisymmetric about the equator, while the second harmonic induced by interaction of the motion with an axisymmetric quadrupolelike field is symmetric. The presence of significant contributions of both harmonics accounts for the observed asymmetries. Note that we only show field vectors where 


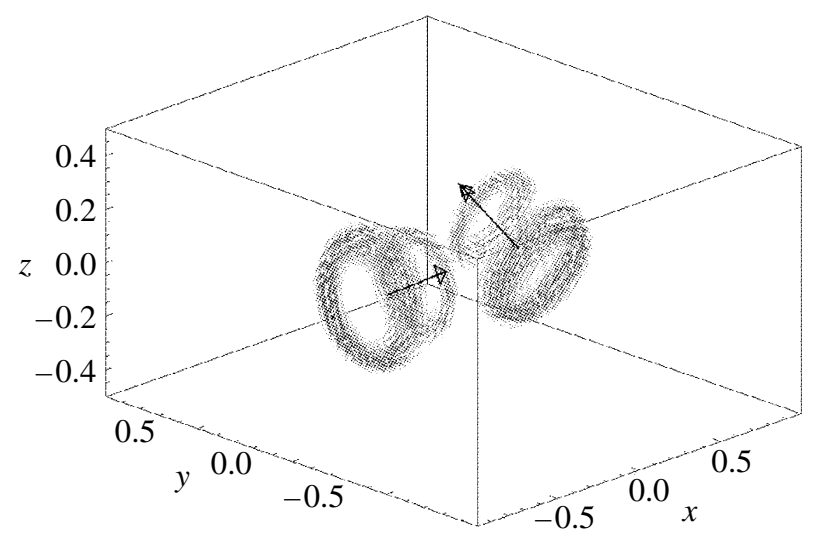

Figure 3. Magnetic field vectors plotted in the three-dimensional space. Vectors are only plotted where the field strength exceeds $80 \%$ of the maximum. Note that the magnetic field takes the form of magnetic flux rings, with fields in opposite senses, around the spinning spheres. The two long arrows indicate the location and orientation of the spin axes of the spheres. $a=0.25$, $d=0.40, R_{\mathrm{m}}=633, \varphi=125^{\circ}$.

Table 3. Comparison of the marginal values of $R_{\mathrm{m}}$ for the primary mode with $\rho=0$ and the secondary mode with $\rho=1$ for two different values of a

(Resolution $31 \times 31 \times 16, L_{z}=\frac{1}{2} L, d=0.40, \varphi=125^{\circ}$.)

\begin{tabular}{ccccccc}
\hline$a$ & $\rho$ & $C_{\omega}$ & $R_{\mathrm{m}}$ & $(3.1)$ & $(\mathrm{A} 19)$ \\
\hline 0.30 & 0 & 3740 & 337 & 265 & 341 \\
0.30 & 1 & 4640 & 418 & 265 & 341 \\
0.35 & 0 & 1500 & 167 & 215 & 184 \\
0.35 & 1 & 2130 & 261 & 215 & 184 \\
\hline
\end{tabular}

the field strength is above a certain threshold. In general, although field is concentrated near the spheres, field lines necessarily extend from one sphere to the other.

\section{(d) A second mode}

Solutions to equation (2.5) with our boundary conditions have the symmetries

$$
\left(B_{x}, B_{y}, B_{z}\right)(-x,-y, z)=(-1)^{\rho}\left(-B_{x},-B_{y}, B_{z}\right)(x, y, z), \quad(\rho=0 \text { or } 1) .
$$

The initial condition and solutions discussed above correspond to the case $\rho=0$. (Note that $\boldsymbol{u}$ has the symmetry $\rho=0$ ) By using as initial condition

$$
A_{z}=\exp \left[-\left(r / r_{0}\right)^{2}-\left(z / z_{0}\right)^{2}\right] \cos \theta,
$$

where $\tan \theta=y / x$, we were able to find the other mode with $\rho=1$. We found numerically that this second mode is typically less preferred, see table 3 . The asymptotic results (3.1) and (A 19) however yield the same marginal values of $R_{\mathrm{m}}$ for $\rho=0$ and 1; see the Appendix. This means that the splitting of the eigenvalue $R_{\mathrm{m}}$ is a small order effect, which is not captured by the asymptotics. Indeed the magnitude of that split, in itself, provides a measure of the departure of the numerics from the asymptotic regime.

Proc. R. Soc. Lond. A (1998) 
Table 4. Marginal values of $C_{\omega}$ and $R_{\mathrm{m}}$ for $a=0.30, d=0.35$ and different angles $\varphi$

\begin{tabular}{rrrrl}
\hline$\varphi$ & $C_{\omega}$ & $R_{\mathrm{m}}$ & $\operatorname{Im} \lambda_{1}$ & resolution \\
\hline $50^{\circ}$ & 15100 & 1360 & 108 & $63 \times 63 \times 32$ \\
$85^{\circ}$ & 17200 & 1550 & 65 & $63 \times 63 \times 32$ \\
$125^{\circ}$ & 1900 & 171 & 0 & $31 \times 31 \times 16$ \\
\hline
\end{tabular}

\section{Oscillatory solutions}

We mentioned in $\S 1$ that Lowes \& Wilkinson (1968) found time-dependent solutions for large values of $R_{\mathrm{m}}$ in an experimental version of a dynamo similar to Herzenberg's. Their experimental set-up differs in some significant respects from our numerical model, and it is quite plausible that this is the result of some nonlinear feedback. A nonlinear feedback cannot, of course, be captured by our linear analysis. On the other hand, it is possible in principle that the largest eigenvalue becomes complex for large values of $R_{\mathrm{m}}$. In this section we describe some numerical experiments undertaken to explore these issues.

\section{(a) Numerical results}

We performed calculations for $\varphi=125^{\circ}, a=0.30$ and $d=0.35$, at fairly high Reynolds number $\left(C_{\omega}=40,000\right.$, i.e. $\left.R_{\mathrm{m}}=3600\right)$, but found only real eigenvalues with $\operatorname{Re} \lambda_{1} \approx 240$. There was no indication of oscillatory behaviour. We then tried to verify the asymptotic result that dynamo action is only possible for $90^{\circ}<|\varphi|<180^{\circ}$, so we ran the case $a=0.30, d=0.35$ for angles $\varphi=85^{\circ}$ and $\varphi=50^{\circ}$, again at fairly large Reynolds numbers. To our surprise we found growing solutions, which were now oscillatory. The solutions are spatially and temporarily well behaved and are therefore expected to be numerically reliable despite the large values of $R_{\mathrm{g}}$. The marginal values of $R_{\mathrm{m}}$ are summarized in table 4 . (All those values are well above the value $R_{\mathrm{m}}=77$ used to produce figure 1.)

As in the steady case, the field consists of two toroidal flux belts surrounding the two spheres. However, the relative strengths of the two belts varies with time in the following way. First the upper belt (i.e. in the sense of positive $\boldsymbol{\omega}$ ) strengthens while the lower one weakens; see the first three panels $(t=0.32-0.36)$ of figure 4 . Then the upper belt migrates rapidly downwards to take the position of the originally lower belt; see the fourth and fifth panel $(t=0.38-0.40)$ of figure 4 . Finally, a new belt emerges to take the position of the originally upper belt and so the cycle continues; see the sixth panel $(t=0.42)$ of figure 4 .

Closer inspection of the work of Lowes \& Wilkinson (1968) revealed that they found their oscillatory solutions when $\varphi=45^{\circ}$. This is consistent with our result that oscillatory dynamo action is possible for $0^{\circ}<\varphi<90^{\circ}$. However, given that Herzenberg's original analysis found solutions only for $90^{\circ}<\varphi<180^{\circ}$ the result of Lowes \& Wilkinson (1968) must have been surprising. On the other hand, the geometry of their experimental set-up was already quite different from Herzenberg's original model, because in the experiment the rotors were bounded by air on one side, rather than the conducting medium. This is probably also the reason why in their earlier work (Lowes \& Wilkinson 1963) they found dynamo action even for $\varphi=90^{\circ}$.

Proc. R. Soc. Lond. A (1998) 

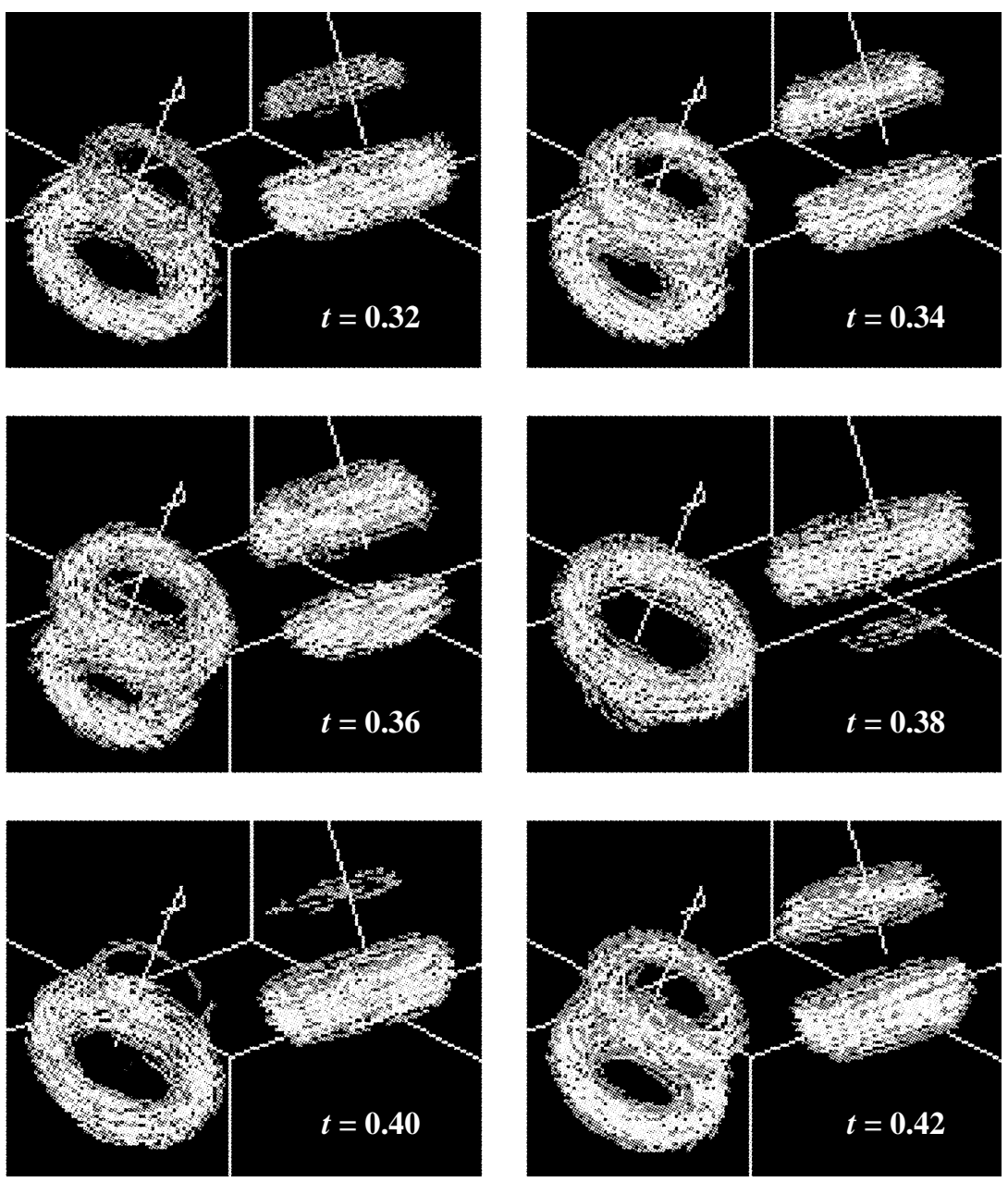

Figure 4. Snapshots of the magnetic field geometry for the oscillatory case covering approximately one cycle. $a=0.30, d=0.35, R_{\mathrm{m}}=1360, \varphi=50^{\circ}$.

\section{(b) Time-dependent asymptotics}

In order to understand the occurrence of oscillatory solutions we now discuss the asymptotic theory in more detail for the marginal case $\operatorname{Re} \lambda_{1}=0$, restricting ourselves to the case $\operatorname{Im} \lambda_{1}<0$. Our development parallels that of Gailitis (1973). He pointed out that the essential modification to Herzenberg's original analysis for rigidly rotating spheres stems from the fact that everywhere, except on the surfaces of the spheres, where the velocity is discontinuous, the magnetic field satisfies

$$
\left(\nabla^{2}+\frac{\mathrm{i}}{\delta^{2}}\right) \boldsymbol{B}=0
$$

where

$$
\delta=\left(\eta /\left|\lambda_{1}\right|\right)^{1 / 2}
$$

is the skin depth linked to the frequency i $\lambda_{1}$ (equal to $-\operatorname{Im} \lambda_{1}=\left|\lambda_{1}\right|$ in our case). In Herzenberg's steady case, the skin depth is infinite, $\delta=\infty$. Following Gailitis (1973), we assume that the sphere radius $a$ is small, and anticipate that skin depth, 
$\delta$, is comparable to the sphere separation, $d$, specifically

$$
a \ll d, \quad \delta=O(d) .
$$

When the conditions (4.3) are satisfied, the magnetic field on the short $a$-length scale in the neighbourhood of each sphere may be approximated as steady on the corresponding short diffusion time scale $a^{2} / \eta$. Accordingly, many of the steady results given in Moffatt (1978), which are special cases of Gibson \& Roberts (1967), can be used to provide a basis for the oscillatory analysis. Relative to an origin at the centre of one of the spheres, the axisymmetric poloidal part of the magnetic field due to the external sources resulting from the other sphere and the periodic boundary conditions can be expressed in the asymptotic form for small $r$ :

$$
\boldsymbol{B}(\boldsymbol{r})=\nabla \Phi+O\left(r^{2}\right),
$$

where

$$
\Phi=A_{1} r P_{1}(\mu)+a^{-1} A_{2} r^{2} P_{2}(\mu)
$$

and

$$
\mu=\hat{\boldsymbol{r}} \cdot \hat{\boldsymbol{\omega}}, \quad \hat{\boldsymbol{r}}=\boldsymbol{r} / r, \quad \hat{\boldsymbol{\omega}}=\boldsymbol{\omega} / \omega,
$$

(see Gibson \& Roberts 1967, equation (24); also Moffatt 1978, equation (6.59) and (6.60)). Here the values of the complex coefficients are given by

$$
A_{1}=\hat{\boldsymbol{\omega}} \cdot \boldsymbol{B}, \quad A_{2}=\frac{1}{2} a \hat{\boldsymbol{\omega}} \cdot \nabla(\hat{\boldsymbol{\omega}} \cdot \boldsymbol{B}),
$$

as evaluated at the origin $\boldsymbol{r}=0$; see Moffatt (1978, equation (6.61)), but implied in the derivation of Gibson \& Roberts (1967, equation (29) and (30)). Of course, the potential magnetic field (4.4) is only part of the externally produced field. It is, however, that part upon which the rotation of the sphere $r<a$ induces the largest magnetic field in the far field $r \gg a$. Specifically, since toroidal magnetic field has no radial component, there is no field induced from it by the sphere's rotation; furthermore, any additional external non-axisymmetric poloidal magnetic fields lead to contributions with faster decay rates in the far field.

The axisymmetric toroidal field, induced outside the sphere by its rotation in the axisymmetric part of the external field, must satisfy (4.2) and decay at infinity. It has the spatial form

$$
\boldsymbol{B}=\hat{\boldsymbol{\omega}} \times \hat{\boldsymbol{r}} \sum_{n=1}^{\infty} B_{n}\left(\frac{\pi}{2 Z}\right)^{1 / 2} K_{n+1 / 2}(Z) \frac{\mathrm{d} P_{n}}{\mathrm{~d} \mu}(\mu), \quad(r>a),
$$

where the $B_{n}$ are complex constants and

$$
Z=\left(\frac{1-\mathrm{i}}{\sqrt{ } 2}\right) \frac{r}{\delta}
$$

and

$$
\left(\frac{\pi}{2 Z}\right)^{1 / 2} K_{n+1 / 2}(Z)=\frac{1}{2} \pi(-Z)^{n}\left(\frac{1}{Z} \frac{\mathrm{d}}{\mathrm{d} Z}\right)^{n} \frac{\mathrm{e}^{-Z}}{Z},
$$

is the modified spherical Bessel function of the third kind. Close to the sphere, where $|Z| \ll 1$, the steady approximation can again be made and the solution can be matched with equation (28) of Gibson \& Roberts (1967) (see also Gibson (1968a, equation (29)) and Moffatt (1978, equation (6.62) but with an erroneous factor $\frac{2}{5}$ to 
be replaced by $\left.\frac{2}{15}\right)$. It gives the result

$$
\boldsymbol{B}=R_{\mathrm{m}} \hat{\boldsymbol{\omega}} \times \hat{\boldsymbol{r}}\left\{-\frac{1}{5} A_{1} \mu\left(\frac{a}{r}\right)^{3}\left(1+Z+\frac{1}{3} Z^{2}\right)+\frac{2}{15} A_{2}\left(\frac{a}{r}\right)^{2}(1+Z)\right\} \mathrm{e}^{-Z},
$$

(see Gailitis 1973, equation (18)), where the terms neglected are smaller by a factor of order $a / d$.

Whereas our interest in (4.11) stems from concern about the nature of solutions when the right-hand side of (3.1) is negative, Gailitis (1973) was essentially concerned about the validity of (3.1) when the right-hand side is actually positive, see the discussion in $\S 5$.

When the sphere separation is small compared with the size of the periodicity box, specifically

$$
d \ll L,
$$

the influence of the remote boundary conditions is negligible and only the direct inductive interaction between the two spheres need be considered. Accordingly, the field (4.11) induced by one sphere is used as the external source field for the other. With the corresponding modifications to Moffatt's (1978) development, which is adequate for our special geomtry, the steady result (3.1) becomes

$$
R_{\mathrm{m}}^{-2}=-\frac{1}{4800}\left(\frac{a}{d}\right)^{6}\left(1+Z_{d}+\frac{1}{3} Z_{d}^{2}\right)\left(1+Z_{d}\right) \mathrm{e}^{-2 Z_{d}} \sin ^{2} \varphi \cos \varphi
$$

where

$$
Z_{d}=(1-i) \chi, \quad \chi=\sqrt{2} d / \delta(>0)
$$

The vanishing of the real part of (4.13) gives $\chi$ as the positive solutions of

$$
\frac{\tan 2 \chi}{2 \chi}=\frac{(3+\chi)(1+\chi)}{3+6 \chi-2 \chi^{3}} .
$$

For those values, the critical value of $R_{\mathrm{m}}$ defined by (4.13) is given by

$$
R_{\mathrm{m}}^{-2}=-\frac{1}{4800}\left(\frac{a}{d}\right)^{6} \mathcal{F}(\chi) \sin ^{2} \varphi \cos \varphi
$$

where

$$
\mathcal{F}(\chi)=\frac{2 \chi(3+\chi)(1+\chi)}{3 \sin 2 \chi} \mathrm{e}^{-2 \chi} .
$$

Whereas steady modes occur for $\chi=0, \mathcal{F}=1$ when $\frac{1}{2} \pi<|\varphi|<\pi$, the oscillatory modes are determined by the first positive root of (4.15), which occurs at $\chi=2.416$ between $\frac{1}{2} \pi$ and $\frac{3}{4} \pi$ giving $\mathcal{F}=-0.239$, and lies in the complementary range $0<|\varphi|<\frac{1}{2} \pi$. In figure 5 we show the dependence of the marginal values of $R_{\mathrm{m}}$ on $\varphi$ for both the steady and the oscillatory branches. Note that, since $\delta=(\sqrt{2} / \chi) d=0.585 d$, the frequency $\left|\operatorname{Im} \lambda_{1}\right|=\eta / \delta^{2}$ is independent of $a$ and $\varphi$.

For the oscillatory modes the agreement between the numerical results and the asymptotic theory is rather bad; see table 5 . This is probably because in our simulations we have $a / \delta \approx 3$, in sharp contrast to the condition (4.3). However the main purpose of carrying out the asymptotics was not to reproduce the numerical results, but rather to gain some understanding of the nature of the oscillatory modes.

Proc. R. Soc. Lond. A (1998) 


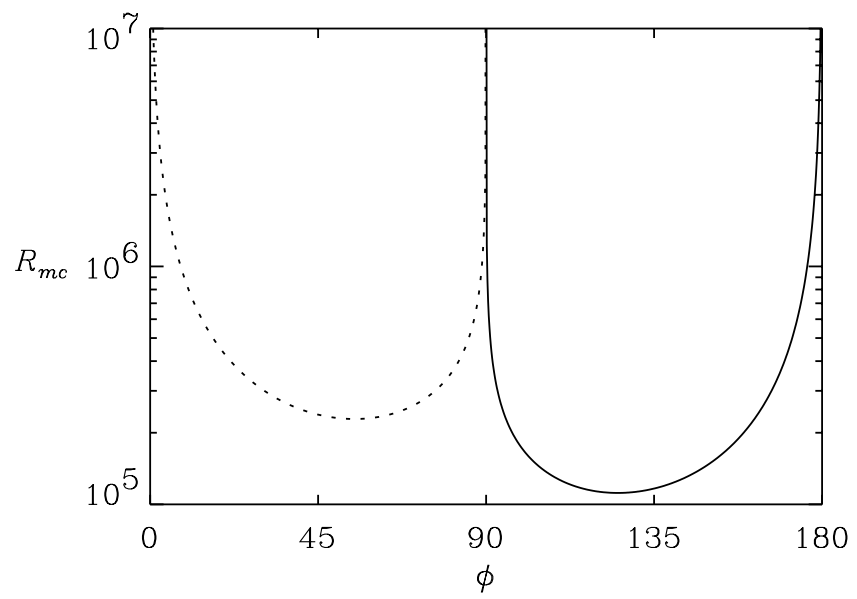

Figure 5. Marginal values of $R_{\mathrm{m}}$ versus $\varphi$ as obtained from the asymptotic theory (assuming here $a / d=0.1)$. The dotted line denotes the new oscillatory branch.

Table 5. Comparison of the asymptotic results (4.16) and (A 19) with the numerical results (Resolution $63 \times 63 \times 32, a=0.30, d=0.35$. The values of $\delta(4.16)$ and $\delta(\mathrm{A} 19)$ follow from the requirement that $R_{\mathrm{m}}$ (4.16) and $R_{\mathrm{m}}$ (A 19), respectively, are real.)

\begin{tabular}{ccccccc}
\hline$\varphi$ & $R_{\mathrm{m}}$ & $\delta$ & $R_{\mathrm{m}}(4.16)$ & $\delta(4.16)$ & $R_{\mathrm{m}}(\mathrm{A} 19)$ & $\delta(\mathrm{A} \mathrm{19)}$ \\
\hline $50^{\circ}$ & 1360 & 0.10 & 366 & 0.20 & 369 & 0.20 \\
$85^{\circ}$ & 1550 & 0.12 & 765 & 0.20 & 769 & 0.20 \\
\hline
\end{tabular}

\section{A comment on the asymptotic theory}

The difficulties mentioned in $\S 1$ originate from Herzenberg's (1958) original treatment of the asymptotic problem. His condition for steady solutions leads to a repeated root for the magnetic Reynolds number $R_{\mathrm{m}}$. This is manifest in his asymptotic treatment as $\left(R_{\mathrm{m}}-\text { whatever }\right)^{2}=$ 'something small'; the difficulty that confronted Herzenberg was what happens if 'something small' is negative? Gibson (1968a) attempted to evaluate that small term but found that the difficulty re-emerged at the next order of approximation. Gailitis (1973), on the other hand, showed that if 'something small' happened to be negative then a marginal mode would exist but oscillate with a low frequency. In so doing he identified the source of the repeated root; it corresponds asymptotically to two distinct modes with different symmetries, as was also pointed out by Moffatt (1978). In our problem we have identified two such classes of solution satisfying the entire system of equations and boundary conditions. On the one hand, the asymptotic solution developed in the Appendix, shows, like Herzenberg, that the repeated root property persists for our more complex system and, as Gailitis, that it is associated with two modes, one from each symmetry class. On the other hand, our numerical solution shows that the values of the marginal magnetic Reynolds numbers for the two modes are actually distinct, contrary to Gibsons \& Robert's (1967) implied speculation. Our result also highlights the origin of the difficulty, associating it (as did Moffatt) with the existence of the two symmetry classes of the full problem and not simply to asymptotic approximations of it; they do not exist for example in Gibson's (1968b) three-rotor assembly.

Proc. R. Soc. Lond. A (1998) 


\section{Discussion}

The Herzenberg dynamo provided the first analytical proof that dynamo action with steady laminar flows is possible. We have found a numerical solution in a situation that closely resembles that of Herzenberg. The major physical difference is that we solve in a box, with periodic boundary conditions in the $x, y$ directions, rather than in a spherical volume surrounded by vacuum. Thus, in reality we have a doubly infinite set of pairs of rotating spheres. For practical reasons, we are limited to rather large values of $a / d$. Nevertheless, we find that the field is strongly localized in the vicinity of the spheres, and the field near the boundaries of our computational box is relatively small. For example, for $a=0.25, d=0.4$, the magnetic energy density between the rotors at $\boldsymbol{x}=(0,0,0)$ is 14 times larger than on the computational boundary at $\boldsymbol{x}=( \pm L, 0,0)$, and, in turn, a typical energy density in one of the field belts is about 15 times larger than that at $\boldsymbol{x}=(0,0,0)$. Consistent with this, we find results that are remarkably similar to those of Herzenberg for the limit of small $a / d$. Figure 2 shows the relation $R_{\mathrm{mc}} \propto(a / d)^{-3}$ (cf. Moffatt 1978, p. 128) to hold approximately over a wide range of $a / d$, and to approach the asymptotic limit more closely for smaller values of $a$ and $d$. Also, the expected $\varphi$ dependence, $R_{\mathrm{mc}}^{2} \propto\left(\sin ^{2} \varphi \cos \varphi\right)^{-1}$, is suggested by the discussion of $\S 3 a$, although we did not systematically determine the marginal values of $R_{\mathrm{m}}$ as a function of $\varphi$. Of course, our model works in a periodic box, rather than the conducting spherical volume of Herzenberg (1958). We regard this essentially as being a computational device, rather than a fundamental distinction. We thus feel that we truly have produced an approximation to Herzenberg's model.

Additionally, we have found a new class of oscillatory solutions for angles $|\varphi|<90^{\circ}$, which was not predicted by Herzenberg's original analysis. It is therefore clear now that dynamo action is possible for all angles $\varphi$, except $\varphi=0^{\circ}, \pm 90^{\circ}$ and $\pm 180^{\circ}$.

Herzenberg discussed his solutions in the context of the Earth's dynamo, although he did not suggest that motions of the type studied (i.e. rotating spheres) would actually be present in the earth's core. Nevertheless, similar motions may occur locally in turbulent flows, which are often thought to consist of turbulent eddies. This comparison is obviously inexact because turbulence is essentially time dependent. What makes this analogy at least superficially attractive, however, is the fact that the magnetic fields generated in the neighbourhood of turbulent eddies or vortex tubes do show some resemblance to the field structure found in the present work. In fact, the magnetic field topology of the Herzenberg dynamo appears to have been rather obscure in the past. (Moffatt (1978) wrote that 'it would be difficult to portray the full three-dimensional field pattern'.) The three-dimensional computer visualization presented here shows that the field consists of magnetic flux rings wrapped round the spinning spheres. This is similar to what has been observed in turbulent (convective) dynamos where magnetic flux tubes are seen to be wrapped round swirling downdraft motions (Nordlund et al. 1992; Brandenburg et al. 1996). Whether or not such analogies make sense needs to be explored. If there is indeed some connection it would be interesting to find out whether there could be some mechanism that could lead to optimal alignment angles between the spin axes of two vortices.

Note in this connection that turbulence consists of vortex tubes that are quasicylindrical rather than spherical in shape. Indeed, Lowes \& Wilkinson $(1963,1968)$ showed using a laboratory experiment that dynamo action is also possible when the two spheres are replaced by rotating cylinders (see also Gailitis 1973). This

Proc. R. Soc. Lond. A (1998) 
model may be more directly relevant to understanding dynamo action associated with nearby vortex tubes in turbulence.

A Herzenberg-like dynamo may also work in detached binary stars. This possibility has been discussed by Dolginov \& Urpin (1979), who gave arguments that certain binary stars (e.g. U Geminorum and similar systems, and symbiotic binaries) may be immersed in a conducting environment. (It may be that the analysis should be modified to allow the magnetic field between the components to be force free.) Obviously, the separation of the stars has to be large enough that the rotation of the two stars is not synchronized with the orbital rotation. In order to estimate the maximum separation below which dynamo action is still possible we consider a binary with components of one solar radius, so $a=7 \times 10^{10} \mathrm{~cm}$. We assume that their angular velocity is anywhere between the solar value and the breakup velocity, so $\omega=3 \times 10^{-6}-7 \times 10^{-4} \mathrm{~s}^{-1}$. Taking temperatures in the range $10^{4}-10^{6} \mathrm{~K}$ the Spitzer conductivity leads to values of the magnetic diffusivity in the range $\eta=10^{4}$ $10^{7} \mathrm{~cm}^{2} \mathrm{~s}^{-1}$. Thus $R_{\mathrm{m}}$ is in the range $10^{9}-10^{15}$. Assuming that the angle $\varphi$ is optimal, dynamo action is possible when the ratio of semi-separation to radius satisfies $d / a<0.2 \times R_{\mathrm{m}}^{1 / 3} \approx 20-20,000$. This condition is easily satisfied, so the Herzenberg dynamo might, in principle, operate in a wide range of detached binaries (provided that tidal torques have not forced alignment of spin axes and/or spin-orbit synchronization). Only for perfectly aligned, or perfectly anti-aligned, or perpendicular, orientations of the spin axes is dynamo action impossible. This mechanism is of particular interest when the stars themselves do not possess intrinsic sources of magnetic field, if considered in isolation.

The Herzenberg dynamo is linear and therefore the magnetic field will eventually be so strong that nonlinear effects will become important and limit the magnetic field strength. There are various feedback mechanisms that can be envisaged: alignment of spin axes, increase of separation, shrinkage or just spindown of the spheres. In the case of binaries, spindown seems most plausible (although this would need a dynamo growth time substantially shorter than the evolutionary timescale for the stars), whereas in the case of vortex tubes mutual alignment could be an important mechanism.

If the Herzenberg dynamo really operates in detached binaries we might expect that spindown and alignment could lead to observable effects. This would be remarkable, because the Herzenberg dynamo is a 'slow' dynamo, which are normally not thought to be important in astrophysics (because they are slow!). Indeed, their $e$ folding time is $\mathcal{O}\left(R_{\mathrm{m}}\right)$ times the orbital period, which can easily become comparable to the lifetime of these stars. Similar problems have been encountered earlier when discussing laminar dynamos in the context of stars. For example the Gailitis dynamo works with meridional circulation and might therefore operate in a wide variety of stars without significant outer convection zones (Moss 1990), but again this mechanism is probably of only marginal importance for the most rapidly rotating magnetic stars of the upper main sequence. In the case of the Herzenberg dynamo however, because of the sensitive $(a / d)^{3}$ dependence of the growth rate, the answer is not yet clear. The common feature of the Herzenberg and Gailitis dynamos is that there are two separate sources of induction, and in the analytic analyses the higher order magnetic field components are assumed to decay before diffusing from one centre of induction to the other. It is now clear that this condition is analytically and conceptually helpful, but certainly not necessary.

We are grateful to Paul Roberts for his illuminating remarks and interesting historical recollections, which have helped us place the present study in context. We thank Frank Lowes for

Proc. R. Soc. Lond. A (1998) 
comments on the manuscript. This work was in part supported by the EC Human Capital and Mobility Networks grant 'Late type stars: activity, magnetism, turbulence' no. ERBCHRXCT940483 and the NATO grant CRG1530959.

\section{Appendix A. Spatial periodicity and reflectional symmetry}

In addition to the spatial periodicity

$$
\boldsymbol{B}(x+2 L, y, z)=\boldsymbol{B}(x, y+2 L, z)=\boldsymbol{B}(x, y, z),
$$

in the $x, y$-plane, we assume that the magnetic field has one of the two rotational invariances $(\rho=0$ or 1$)$, see equation (3.2), and the reflectional symmetry

$$
\left(B_{x}, B_{y}, B_{z}\right)\left(x, y, 2 L_{z}-z\right)=\left(-B_{x},-B_{y}, B_{z}\right)(x, y, z),
$$

compatible with the boundary conditions on $z= \pm L_{z}$. Magnetic field with this symmetry can be achieved in an unbounded domain with rotors of radius $a$ and angular velocity

$$
\omega_{\alpha}=\omega \hat{\boldsymbol{\omega}}_{\alpha}, \quad \hat{\boldsymbol{\omega}}_{\boldsymbol{\alpha}}=\left(0,(-1)^{n+\sigma} \sin \frac{1}{2} \varphi, \cos \frac{1}{2} \varphi\right),
$$

with centres

$$
\boldsymbol{x}_{\boldsymbol{\alpha}}=L \boldsymbol{\xi}_{\boldsymbol{\alpha}}, \quad \boldsymbol{\xi}_{\boldsymbol{\alpha}}=\left((-1)^{\sigma} D+2 l, 2 m, 2 n \Delta\right), \quad \boldsymbol{\alpha}=(\sigma, l, m, n):
$$

$\sigma=0$ and $1, l, m, n$ are integers; $D=d / L$ and $\Delta=L_{z} / L$. Below we consider results valid in the Herzenberg (1958) limit

$$
a \ll d .
$$

With the above symmetries and rotor orientations, the constants $A_{1}^{\alpha}, A_{2}^{\alpha}$ defining the local magnetic field (4.4) at $\boldsymbol{x}_{\boldsymbol{\alpha}}$ are given by

$$
A_{1}^{\alpha}=(-1)^{\rho \sigma} A_{1}, \quad A_{2}^{\alpha}=(-1)^{\rho \sigma+n} A_{2},
$$

in terms of only two constants $A_{1}$ and $A_{2}$. We calculate their values at $\boldsymbol{x}_{\mathbf{0}}=(d, 0,0)$, corresponding to $\boldsymbol{\alpha}=\mathbf{0}=(0,0,0,0)$, using Moffatt (1978, equations (6.64) and $(6.65))$. The result is

$$
\left(\begin{array}{c}
A_{1} \\
A_{2}
\end{array}\right)=\frac{1}{5}\left(\frac{a}{d}\right)^{3} R_{\mathrm{m}}\left(\begin{array}{cc}
\mathcal{P}_{11} & \frac{2 d}{a} \mathcal{P}_{12} \\
\frac{a}{2 d} \mathcal{P}_{21} & \mathcal{P}_{22}
\end{array}\right)\left(\begin{array}{c}
A_{1} \\
A_{2}
\end{array}\right)
$$

where

$$
\begin{aligned}
& \mathcal{P}_{11}=-D^{3} \sum_{\boldsymbol{\alpha} \neq \mathbf{0}}(-1)^{\rho \sigma} \frac{\left(\hat{\boldsymbol{\omega}}_{\boldsymbol{\alpha}} \cdot \boldsymbol{\zeta}_{\boldsymbol{\alpha}}\right)}{\zeta_{\boldsymbol{\alpha}}^{5}}\left(\hat{\boldsymbol{\omega}}_{\mathbf{0}} \times \hat{\boldsymbol{\omega}}_{\boldsymbol{\alpha}}\right) \cdot \boldsymbol{\zeta}_{\boldsymbol{\alpha}} \\
& \mathcal{P}_{12}=\frac{1}{3} D^{2} \sum_{\boldsymbol{\alpha} \neq \mathbf{0}}(-1)^{\rho \sigma+n} \frac{\left(\hat{\boldsymbol{\omega}}_{\mathbf{0}} \times \hat{\boldsymbol{\omega}}_{\boldsymbol{\alpha}}\right) \cdot \boldsymbol{\zeta}_{\boldsymbol{\alpha}}}{\zeta_{\boldsymbol{\alpha}}^{3}} \\
& \mathcal{P}_{21}=-D^{4} \sum_{\boldsymbol{\alpha} \neq \mathbf{0}}(-1)^{\rho \sigma}\left[\frac{\left(\hat{\boldsymbol{\omega}}_{\mathbf{0}} \cdot \hat{\boldsymbol{\omega}}_{\boldsymbol{\alpha}}\right)}{\zeta_{\boldsymbol{\alpha}}^{5}}-5 \frac{\left(\hat{\boldsymbol{\omega}}_{\mathbf{0}} \cdot \boldsymbol{\zeta}_{\boldsymbol{\alpha}}\right)\left(\hat{\boldsymbol{\omega}}_{\boldsymbol{\alpha}} \cdot \boldsymbol{\zeta}_{\boldsymbol{\alpha}}\right)}{\zeta_{\boldsymbol{\alpha}}^{7}}\right]\left(\hat{\boldsymbol{\omega}}_{\mathbf{0}} \times \hat{\boldsymbol{\omega}}_{\boldsymbol{\alpha}}\right) \cdot \boldsymbol{\zeta}_{\boldsymbol{\alpha}} \\
& \mathcal{P}_{22}=-D^{3} \sum_{\boldsymbol{\alpha} \neq \mathbf{0}}(-1)^{\rho \sigma+n} \frac{\left(\hat{\boldsymbol{\omega}}_{\mathbf{0}} \cdot \boldsymbol{\zeta}_{\boldsymbol{\alpha}}\right)}{\zeta_{\boldsymbol{\alpha}}^{5}}\left(\hat{\boldsymbol{\omega}}_{\mathbf{0}} \times \hat{\boldsymbol{\omega}}_{\boldsymbol{\alpha}}\right) \cdot \boldsymbol{\zeta}_{\boldsymbol{\alpha}}
\end{aligned}
$$

Proc. R. Soc. Lond. A (1998) 
with

Explicit values are

$$
\zeta_{\alpha}=\left|\zeta_{\alpha}\right|, \quad \zeta_{\alpha}=\xi_{0}-\xi_{\alpha}
$$

$$
\begin{gathered}
\boldsymbol{\zeta}_{\alpha}=-\left(\left[(-1)^{\sigma}-1\right] D+2 l, 2 m, 2 n \Delta\right), \\
\hat{\boldsymbol{\omega}}_{\mathbf{0}} \cdot \boldsymbol{\zeta}_{\alpha}=-2 m \sin \frac{1}{2} \varphi-2 n \Delta \cos \frac{1}{2} \varphi, \\
\hat{\boldsymbol{\omega}}_{\alpha} \cdot \boldsymbol{\zeta}_{\alpha}=(-1)^{\sigma+n+1} 2 m \sin \frac{1}{2} \varphi-2 n \Delta \cos \frac{1}{2} \varphi, \\
\hat{\boldsymbol{\omega}}_{0} \cdot \hat{\boldsymbol{\omega}}_{\alpha}=(-1)^{\sigma+n} \sin ^{2} \frac{1}{2} \varphi+\cos ^{2} \frac{1}{2} \varphi, \\
\left(\hat{\boldsymbol{\omega}}_{\mathbf{0}} \times \hat{\boldsymbol{\omega}}_{\alpha}\right) \cdot \boldsymbol{\zeta}_{\alpha}=\frac{1}{2}\left[(-1)^{\sigma+n}-1\right]\left\{\left[(-1)^{\sigma}-1\right] D+2 l\right\} \sin \varphi ;
\end{gathered}
$$

all independent of the ratio $a / d$. Under the transformation $(\sigma, l, m, n) \rightarrow(\sigma, l$, $-m,-n)$, the signs of $\hat{\omega}_{0} \cdot \zeta_{\alpha}$ and $\hat{\omega}_{\alpha} \cdot \zeta_{\alpha}$ change, those of $\zeta_{\alpha}, \hat{\omega}_{0} \cdot \hat{\omega}_{\alpha}$ and $\left(\hat{\omega}_{0} \times \hat{\omega}_{\alpha}\right) \cdot \zeta_{\alpha}$ are unaltered, while all magnitudes remain the same. With one exception the same holds for the particular case $\sigma=0$ under the transformation $(0, l, m, n) \rightarrow(0,-l, m, n)$; the exception is that now $\left(\hat{\boldsymbol{\omega}}_{0} \times \hat{\boldsymbol{\omega}}_{\boldsymbol{\alpha}}\right) \cdot \boldsymbol{\zeta}_{\boldsymbol{\alpha}}$ changes sign instead of keeping the same sign. As a consequence of the former symmetries, we have upon summation that

$$
\mathcal{P}_{11}=\mathcal{P}_{22}=0
$$

It follows from (A 7) that

$$
R_{\mathrm{m}}^{-2}=\frac{1}{25}\left(\frac{a}{d}\right)^{6} \mathcal{P}_{12} \mathcal{P}_{21}, \quad(\mathcal{P}=\mathcal{P}(D, \Delta, \varphi)),
$$

where we emphasize that $\mathcal{P}$ is a function of $D, \Delta$ and $\varphi$ alone. As a consequence of the latter symmetries, all $\sigma=0$ terms in the summations cancel, leaving only the $\sigma=1$ terms proportional to $(-1)^{\rho}$. So under the change of $\rho$, namely $0 \rightarrow 1$, we have $\mathcal{P} \rightarrow-\mathcal{P}, R_{\mathrm{m}}$ remains unchanged and $\left(A_{1}, A_{2}\right) \rightarrow\left(A_{1},-A_{2}\right)$. It means that the eigenvalue $R_{\mathrm{m}}$ is repeated with two distinct eigenvectors $\boldsymbol{B}(\boldsymbol{x})$ corresponding to the distinct symmetries (3.2) $\rho=0$ and 1 .

Moffatt's result equation (3.1) is recovered in the large box size limit, for which only the single terms identified by $\boldsymbol{\alpha}=(1,0,0,0)$ are retained giving

$$
\mathcal{P}_{12} \mathcal{P}_{21} \sim-\frac{1}{192} \sin ^{2} \varphi \cos \varphi, \quad \text { as } D \uparrow \infty,
$$

at fixed $\Delta$ and $\varphi$ independent of $\rho$. Close inspection of (A 9) and (A 10) shows however that the values of $R_{\mathrm{m}}$ are the same in those two cases.

In table 2 we compare the asymptotic results (3.1) and (A 19) with the numerical results. It turns out that the marginal values of $R_{\mathrm{m}}$ converge in an oscillatory fashion between even and odd truncation levels $N$. Therefore we averaged the results for even and odd truncation level. The results presented in the table are for $N=10$, but comparison with $N=8$ gave the same result in all cases. In general the agreement with the numerical results is now improved, except for cases where $a / d$ is very close to unity.

Equation (A 19) also generalizes (4.13), which requires $a \ll \delta$ (see (4.3)) in addition to (A 5) for its validity. Note, however, that the $\mathcal{P}$-matrix is modified and the key coefficients $\mathcal{P}_{12}$ and $\mathcal{P}_{21}$ are replaced by

$$
\mathcal{P}_{12}=\frac{1}{3} D^{2} \sum_{\boldsymbol{\alpha} \neq \mathbf{0}}(-1)^{\rho \sigma+n} \frac{\left(\hat{\boldsymbol{\omega}}_{\mathbf{0}} \times \hat{\boldsymbol{\omega}}_{\boldsymbol{\alpha}}\right) \cdot \boldsymbol{\zeta}_{\boldsymbol{\alpha}}}{\zeta_{\boldsymbol{\alpha}}^{3}}\left(1+Z_{\boldsymbol{\alpha}}\right) \mathrm{e}^{-Z_{\boldsymbol{\alpha}}},
$$

Proc. R. Soc. Lond. A (1998) 


$$
\begin{aligned}
\mathcal{P}_{21}= & -D^{4} \sum_{\boldsymbol{\alpha} \neq \mathbf{0}}(-1)^{\rho \sigma}\left[\frac{\left(\hat{\boldsymbol{\omega}}_{\mathbf{0}} \cdot \hat{\boldsymbol{\omega}}_{\boldsymbol{\alpha}}\right)}{\zeta_{\boldsymbol{\alpha}}^{5}}\left(1+Z_{\boldsymbol{\alpha}}+\frac{1}{3} Z_{\boldsymbol{\alpha}}^{2}\right)\right. \\
& \left.-\frac{\left(\hat{\boldsymbol{\omega}}_{\mathbf{0}} \cdot \boldsymbol{\zeta}_{\boldsymbol{\alpha}}\right)\left(\hat{\boldsymbol{\omega}}_{\boldsymbol{\alpha}} \cdot \boldsymbol{\zeta}_{\boldsymbol{\alpha}}\right)}{\zeta_{\boldsymbol{\alpha}}^{7}}\left(5+5 Z_{\boldsymbol{\alpha}}+2 Z_{\boldsymbol{\alpha}}^{2}+\frac{1}{3} Z_{\boldsymbol{\alpha}}^{3}\right)\right]\left(\hat{\boldsymbol{\omega}}_{\mathbf{0}} \times \hat{\boldsymbol{\omega}}_{\boldsymbol{\alpha}}\right) \cdot \boldsymbol{\zeta}_{\boldsymbol{\alpha}} \mathrm{e}^{-Z_{\boldsymbol{\alpha}}},
\end{aligned}
$$

where

$$
Z_{\boldsymbol{\alpha}}=\left(\frac{1-\mathrm{i}}{\sqrt{ } 2}\right) \frac{L \zeta_{\boldsymbol{\alpha}}}{\delta}
$$

To obtain the critical Reynolds number and frequency, equate real and imaginary parts as before. Of course, the result (4.13) is recovered in the large box limit $D \uparrow \infty$ (at fixed $\Delta$ ).

\section{References}

Backus, G. E. 1958 A class of self-sustaining dissipative spherical dynamos. A. Phys. 4, 372-447.

Brandenburg, A., Nordlund, A., Stein, R. F. \& Torkelsson, U. 1995 Dynamo generated turbulence and large scale magnetic fields in a Keplerian shear flow. Atrophys J. 446, 741-754.

Brandenburg, A., Jennings, R. L., Nordlund, Å., Rieutord, M., Stein, R. F. \& Tuominen, I. 1996 Magnetic structures in a dynamo simulation. J. Fluid Mech. 306, 325-352.

Childress, S. 1983 Stationary induction by intermittent velocity fields. In Stellar and planetary magnetism. (ed. A. M. Soward), pp. 81-90. London: Gordon \& Breach.

Dolginov, A. Z. \& Urpin, V. A. 1979 The inductive generation of the magnetic field in binary systems. Astron. Astrophys. 79, 60-69.

Gailitis, A. K. 1970 Self-excitation of a magnetic field by a pair of annular vortices. Magn. Gidrodin. (Engl. transl. Magnetohydrodynam. 6, 14-17.)

Gailitis, A. K. 1973 On the theory of Herzenberg's dynamo. Magn. Gidrodin. 4, 12-16.

Gibson, R. D. $1968 a$ The Herzenberg dynamo. I. Q. J. Mech. Appl. Math. 21, 243-255.

Gibson, R. D. $1968 b$ The Herzenberg dynamo. II. Q. J. Mech. Appl. Math. 21, 257-267.

Gibson, R. D. 1969 The Herzenberg dynamo. In The application of modern physics to the Earth and planetary interiors (ed. S. K. Runcorn). London: Wiley.

Gibson, R. D. \& Roberts, P. H. 1967 Some comments on the theory of homogeneous dynamos. In Magnetism in the cosmos (ed. W. R. Hindmarsh, F. J. Lowes, P. H. Roberts \& S. K. Runcorn). Edinburgh: Oliver \& Boyd.

Herzenberg, A. 1958 Geomagnetic dynamos. Phil. Trans. R. Soc. Lond. A 250, 543-583.

Herzenberg, A. \& Lowes, F. J. 1957 Electromagnetic induction in rotating conductors. Phil. Trans. R. Soc. Lond. A 249, 507-584.

Lele, S. K. 1992 Compact finite difference schemes with spectral-like resolution. J. Comp. Phys. 103, $16-42$.

Lowes, F. J. \& Wilkinson, I. 1963 Geomagnetic dynamo: a laboratory model. Nature 198, 11581160.

Lowes, F. J. \& Wilkinson, I. 1968 Geomagnetic dynamo: an improved laboratory model. Nature 219, $717-718$.

Moffatt, H. K. 1978 Magnetic field generation in electrically conducting fluids. Cambridge University Press.

Moss, D. 1990 A Gailitis-type dynamo in the magnetic CP stars? Mon. Notices R. Astr. Soc. 243, 537-542.

Nordlund, Å., Brandenburg, A., Jennings, R. L., Rieutord, M., Ruokolainen, J., Stein, R. F. \& Tuominen, I. 1992 Dynamo action in stratified convection with overshoot. Astrophys. J. 392, $647-652$.

Roberts, P. H. 1967a An introduction to magnetohydrodynamics. London: Longmans.

Proc. R. Soc. Lond. A (1998) 
Roberts, P. H. $1967 b$ The Herzenberg dynamo. In Geophysical fluid dynamics summer study program course lectures (ed. G. Veronis), pp. 86-105. Woods Hole Oceanographic Institute of Technology report. (Notes submitted by R. Thompson.)

Roberts, P. H. 1971 Dynamo theory. In Mathematical problems in the geophysical sciences (ed. W. H. Reid), pp. 129-209. Providence, RI: American Mathematical Society. 